\title{
Philosophiques
}

\section{After phrenology : Neural Reuse and the Interactive Brain de Michael L. Anderson (MIT Press, 2014)}

\section{Mélyssa Thibodeau-Doré et Pierre Poirier}

Volume 43, numéro 2, automne 2016

URI : https://id.erudit.org/iderudit/1038222ar

DOI : https://doi.org/10.7202/1038222ar

Aller au sommaire du numéro

Éditeur(s)

Société de philosophie du Québec

ISSN

0316-2923 (imprimé)

1492-1391 (numérique)

Découvrir la revue

Citer ce compte rendu

Thibodeau-Doré, M. \& Poirier, P. (2016). Compte rendu de [After phrenology: Neural Reuse and the Interactive Brain de Michael L. Anderson (MIT Press, 2014)]. Philosophiques, 43(2), 533-537. https://doi.org/10.7202/1038222ar d'utilisation que vous pouvez consulter en ligne.

https://apropos.erudit.org/fr/usagers/politique-dutilisation/ 


\title{
Comptes rendus
}

\author{
After phrenology: Neural Reuse and the Interactive Brain de Michael \\ L. Anderson (MIT Press, 2014)
}

After phrenology: Neural Reuse and the Interactive Brain, le premier ouvrage de Michael L. Anderson, explore de manière critique puis constructive deux questions centrales des neurosciences cognitives contemporaines: I) l'organisation fonctionnelle du cerveau; et 2) l'appareil conceptuel propre à saisir adéquatement cette organisation. L'ouvrage, que la majorité des commentateurs d'une récente étude critique ${ }^{1}$ ont estimé important - l'un (Silberstein 20I6) allant même jusqu'à le déclarer le meilleur ouvrage du siècle! - conçoit une réponse à ces questions à partir de résultats de recherches récentes, certaines de l'auteur lui-même, provenant d'une forme de la discipline que d'aucuns appellent «neurosciences cognitives 2.0 ». Si nous partageons l'opinion de ces commentateurs quant à l'importance de l'ouvrage, il nous faut insister sur le fait que la réponse proposée est, comme l'auteur lui-même le reconnaît à plusieurs endroits, hautement spéculative et souvent compatible avec une interprétation plus orthodoxe.

Le traitement critique de la question de l'organisation fonctionnelle se veut une attaque contre la pratique actuelle en neurosciences cognitives, où Anderson voit un localisationnisme naif qui n'est pas sans rappeler selon lui la phrénologie du XIX ${ }^{e}$ siècle. Les neurosciences cognitives seraient imprégnées de ce qu'Anderson nomme la théorie compositionnelle et computationnelle de l'esprit (CCTM), qui veut que la cognition soit composée d'un ensemble de mécanismes fonctionnellement spécialisés et localisés dans des régions circonscrites du cerveau. Cette théorie pousse les chercheurs à ne recourir aux méthodes d'imagerie cérébrale que pour associer les modules cognitifs aux régions cérébrales qui les sous-tendraient. À cette "nouvelle phrénologie» (Uttal 200I), Anderson oppose un nouveau paradigme qu'il nomme réutilisation neuronale $(\mathrm{RN})$, lequel permet de repenser les neurosciences cognitives ainsi que de les fonder sur des assises théoriques en continuité avec les théories de la cognition incarnée (embodied). Selon $\mathrm{RN}$, les régions du cerveau ne sont pas fonctionnellement spécialisées mais peuvent être utilisées et réutilisées pour réaliser diverses tâches cognitives, dans différents domaines de la cognition. $\mathrm{RN}$ sert à la fois de modèle de l'architecture cognitive, d'hypothèse de neuro-plasticité à l'œuvre dans le cerveau, et de paradigme méthodologique pour guider la recherche en neurosciences cognitives. RN s'oppose à toutes les

1. Voir le précis de l'ouvrage rédigé par l'auteur, les commentaires critiques et la réponse de l'auteur dans Behavioral and Brain Science (2016).

PHILOSOPHIQUES 43/2 — Automne 2016, p. 533-550 
assises centrales de CCTM: le localisationnisme, la spécialisation fonctionnelle, le computationnalisme classique, voire tout l'appareil conceptuel des sciences cognitives. Aux construits traditionnels hérités de la psychologie du XIX ${ }^{e}$ siècle (perception, attention, mémoire, etc.), reconstruits dans la forme computationnelle que leur a donné les sciences cognitives, il oppose les concepts encore en émergence des sciences cognitives incarnées.

Afin de soutenir RN, Anderson présente trois lignes argumentatives. La première est de nature empirique: les données récoltées en neurosciences lors des dernières décennies montrent que les mêmes régions cérébrales sont impliquées dans plusieurs tâches et pour des domaines cognitifs variés. Anderson examine d'ailleurs de manière extensive, au chapitre I, les études empiriques allant dans ce sens. La seconde ligne argumentative est évolutive. Anderson avance qu'il n'aurait pas été économique pour l'évolution de "construire" dans le cerveau des modules spécialisés pour résoudre chaque nouveau problème adaptatif. Plutôt, elle aurait de préférence sélectionné des cerveaux capables de réutiliser les ressources neuronales déjà existantes, formant de nouveaux partenariats entre les régions cérébrales pour réaliser les tâches demandées. La troisième ligne argumentative consiste à souligner le pouvoir prédictif de RN. Ce paradigme permet de faire trois prédictions quant à l'organisation fonctionnelle du cerveau. D'abord, les mêmes régions cérébrales seront impliquées dans des tâches cognitives diverses. Ensuite, la spécificité des fonctions cognitives dépendra de la configuration des ressources utilisées pour réaliser une tâche et finalement, les fonctions cognitives évolutivement récentes seront réalisées de façon plus largement distribuée dans le cerveau. Anderson présente des évidences empiriques cohérentes avec ces trois prédictions.

Bien que la réutilisation neuronale soit l'hypothèse centrale du livre, Anderson y développe plusieurs autres propositions intéressantes. Il suggère au chapitre II une approche du développement fonctionnel neuronal capable de rendre compte des données du premier chapitre: la différentiation et recherche interactive (interactive differentiation and search, IDS). Selon IDS, les régions cérébrales vont, au cours de l'ontogenèse, développer des profils de réponses distinctifs qui se manifestent par des tendances fonctionnelles régionales. Ces tendances, influencées par des biais corticaux hérités et des facteurs extrinsèques (expérience, relation avec d'autres régions), permettent à la même région de produire des réponses différentes selon les partenariats qu'elle établit avec d'autres régions cérébrales. Une analyse des données suggère toutefois la présence fondamentale d'un processus de recherche, qui sert à tester rapidement de multiples coalitions neuronales pour trouver laquelle est la plus fonctionnellement adéquate pour réaliser une tâche spécifique. Anderson présente des recherches dans divers domaines qui sont compatibles avec l'existence d'un tel mécanisme et suggère ensuite que certains phénomènes liés à la dynamique dendritique ainsi que la transmission volumique (volume transmission) des neurotransmetteurs pourraient servir de base physiologique au mécanisme de recherche. 
Anderson cherche ensuite à développer une conception de l'organisation fonctionnelle du cerveau compatible avec RN. Selon lui, pour réaliser des tâches cognitives, les régions cérébrales s'assemblent en coalitions fonctionnelles, formant ce qu'il nomme des TALoNS (Transiently Assembled Local Neural Subsystems). Ces assemblées neuronales transitoires sont des composantes de réseaux fonctionnels à plus grande échelle. Ainsi, une même région cérébrale peut former différents TALoNS pour participer à diverses coalitions fonctionnelles. Une conséquence intéressante de cette conception est que l'activation seule d'une région cérébrale n'est pas suffisante pour révéler l'état interne des TALoNS. Il faudrait être en mesure d'évaluer l'état de connectivité au sein même d'une région cérébrale d'intérêt afin de connaitre son statut fonctionnel.

Le chapitre IV explore le vocabulaire apte à saisir les tendances fonctionnelles des régions cérébrales et à identifier les facteurs psychologiques neurologiquement pertinents (NRP factors; Neurologically Relevant Psychological Factors) manifestés par leur activité: ce qui sera exprimé en termes de différentes charges (loading) de l'activité des régions cérébrales sur ces facteurs NRP, lesquels, ensemble, capturent le plus de variance possible de l'activité cérébrale. La méthode privilégiée par Anderson pour y parvenir est celle qui donne son titre au chapitre car elle suggère de concevoir les régions cérébrales comme ayant des "personnalités ». Au terme d'analyses statistiques de l'activité globale de cerveaux appliqués à résoudre diverses tâches, l'objectif est de trouver la signature neurologique de la réalisation de diverses tâches, et dans ce contexte la tendance de chaque région, sa personnalité, à participer à ces signatures.

Anderson présente ensuite les recherches et théories de chercheures et chercheurs travaillant dans la perspective de la cognition incarnée. Le but est de montrer que nous pouvons y trouver les ressources théoriques pour aborder les neurosciences cognitives dans une perspective qui appuie RN. Il défend d'abord qu'afin de trouver les facteurs NRP, il faut tenir compte du rôle que joue le cerveau au sein d'un organisme situé dans son environnement. Cela implique selon lui de comprendre le cerveau comme un système de contrôle dynamique responsable de générer l'action. Dans cette perspective, il suggère d'importer le langage des affordances ("potentialités ») dans notre ontologie cognitive. Il soutient également qu'il faut évacuer l'idée selon laquelle la perception est «reconstructive», générant des représentations du monde pour pallier à la pauvreté des stimuli sensoriels. Anderson prétend que la perception est plutôt performative et parvient à générer des stimuli sensoriels riches en ajustant l'action afin de générer des expériences sensorielles révélant, sous la forme d'invariances, la spécificité des objets du monde. De façon plus générale, il défend que toutes les fonctions cognitives sont performatives et que, bien qu'il puisse être approprié de comprendre certains états neuronaux en termes représentationnels, on comprendra mieux la plupart d'entre eux en termes dynamiques. 
Dans les derniers chapitres, Anderson montre comment on peut repenser le computationnalisme à partir de ce qui a été développé précédemment. Au computationnalisme et au connexionnisme classique il oppose un certain type de réseaux de neurones artificiels (des réseaux dynamiques possédant des connexions massivement ré-entrantes à plusieurs niveaux) et une interprétation du travail computationnel qu'ils produisent. Dans ces réseaux, on peut comprendre la présence simultanée de plusieurs patrons d'activation qui se chevauchent comme étant la présence dans le réseau de plusieurs intentions comportementales possibles qui s'influencent simultanément, et la concurrence entre les états comme étant le processus de prise de décision comportementale. Ce mécanisme neurologiquement plausible de computation par concurrence, basé sur la sommation non linéaire, est associé par la suite à l'hypothèse de la concurrence des affordances de Cisek (2007), ce qui l'amène à ajouter un mécanisme de concurrence par inhibition à celui basé sur la sommation non linéaire. Anderson cherchera ensuite à montrer que cette conception vaut également pour la cognition dite supérieure. Il explique d'abord de manière générale comment un modèle basé sur le contrôle et la concurrence pourrait valoir pour la cognition supérieure. Il reprend ici les réflexions de Wells (2006) (et d'autres) selon lesquelles on peut concevoir le ruban d'une machine de Turing comme son environnement. Cette conception de la cognition supérieure où le cerveau joue le rôle d'un contrôleur appliquant les règles d'une table d'instruction d'une machine de Turing, par l'intermédiaire des effecteurs et des senseurs, à des symboles présents dans l'environnement ne représente pas la seule façon de concevoir le rôle de l'environnement et du corps dans la constitution de la cognition supérieure: c'est simplement celle qui cadre le mieux avec RN. La conception est ensuite illustrée dans deux cas particuliers: la cognition mathématique et le langage. En ce qui concerne ce dernier, l'objectif n'est pas de nier la pertinence des travaux plus formels sur le langage mais de montrer qu'ils n'impliquent pas la nécessité de postuler l'évolution (difficile) d'un module spécialisé. L'activité langagière est le genre de chose qu'un cerveau incarné en interaction constante avec son environnement peut déjà faire.

Une proposition intéressante du dernier chapitre, qui sert en quelque sorte de conclusion à l'ouvrage, est que CCTM ne représenterait pas une véritable théorie fonctionnaliste du cerveau. En effet, cette théorie fait abstraction de l'histoire évolutive du cerveau et ignore ainsi sa fonction fondamentale: générer l'action. Pour Anderson, une théorie proprement fonctionnaliste devra adopter trois principes: I) l'architecture fonctionnelle du cerveau a été modelée par la sélection naturelle; 2) notre complexité comportementale émerge de l'habileté du cerveau à former des coalitions fonctionnelles; et 3) le cerveau est orienté vers l'action, sa fonction étant de coordonner l'ajustement d'un organisme à des circonstances externes.

L'ouvrage est ainsi constitué de deux parties: une première vouée à la critique de la conception compositionnelle et modulaire de l'organisation 
cérébrale et à la promotion d'une conception alternative, la réutilisation neuronale; une seconde vouée à la critique de la conception traditionnelle, internaliste et computationnelle de la cognition, et à la promotion d'une conception alternative, la cognition incarnée. Anderson insiste tout au long de l'ouvrage sur le lien qui va de la première partie à la seconde: la réutilisation neuronale est, selon lui, la forme d'architecture cérébrale la plus apte à soutenir une cognition qui soit incarnée, située et énactive. Ce n'est pas la seule cependant. Plus récemment en effet, un ouvrage de Clark (20I6) soutient la même chose en ce qui concerne la forme d'architecture cérébrale au cœur de la conception dite "prédictive» de la cognition. Puisqu'elles concernent des aspects différents de l'organisation cérébrale, ces deux conceptions ne sont pas en concurrence, la prédiction pouvant par exemple être une des déterminantes de la réutilisation. Il serait intéressant de voir si ces deux approches peuvent être combinées en une conception unifiée d'une architecture cérébrale qui soutient la cognition incarnée. Anderson insiste moins cependant sur le lien qui va dans le sens inverse, c'est-à-dire de la perspective incarnée vers la réutilisation neuronale, et nous voudrions l'ébaucher brièvement pour terminer cette revue, car il montre l'unité forte des deux composantes centrales de l'ouvrage. Pour asseoir entièrement son interprétation des recherches en neurosciences cognitives 2.0, Anderson doit établir quel est le moyen impropre à identifier et interpréter les facteurs NRP, car selon lui l'ontologie traditionnelle des sciences cognitives n'est pas appropriée à cette tâche. À son avis, c'est justement à partir des sciences cognitives incarnées, et notamment autour de concepts comme affordance et concurrence d'affordances que l'on pourra développer une ontologie capable de donner un sens aux construits identifiés par les analyses factorielles. Cependant, comme il le reconnaît, beaucoup de travail reste à faire pour constituer ce vocabulaire et le mettre en relation avec les construits neuroscientifiques 2.o. Si on y parvient, la révolution naissante, encore chétive, ébauchée par Anderson renouvellera autant les neurosciences que la psychologie dans une structure conceptuelle (enfin) unifiée.

\section{Références}

Cisek, P. «Cortical Mechanisms of Action Selection: The Affordance Competition Hypothesis". Philosophical Transactions of the Royal Society of London. Series B, Biological Sciences, 2007, 362, I 58 5-I 599.

Silberstein, M. «Open Peer Commentary: The Implications of Neural Reuse for the Future of Both Cognitive Neuroscience and Folk Psychology». Behavioral and Brain Sciences, 39, 2016, 27-29.

Uttal, W. The New Phrenology: The Limits of Localizing Cognitive Processes in the Brain. Cambridge, MA, MIT Press, 200I.

Wells, A. Rethinking Cognitive Computation: Turing and the Science of the Mind. London, Palgrave, 2006.

MÉLYSSA THIBODEAU-DORÉ ET PIERRE POIRIER 